\title{
Optically Switched Arrayed Waveguide Gratings Using Phase Modulation
}

\author{
Soon Thor Lim, Ching Eng Png, Frederic Y. Gardes, and Graham T. Reed
}

\begin{abstract}
This paper reports a theoretical analysis of novel optically switched arrayed waveguide gratings (AWG) based on a silicon-on-insulator (SOI) platform using phase modulation. The phase modulation can be achieved by integrating optical phase modulators across the grating arms, incorporating four-way splitters and combiners to reduce the thermal optic effect. The phase modulator operates by injecting the free carriers to change the refractive index in the guiding region, hence allowing different wavelengths to route to different output waveguides. Also, with appropriate absorption caused by the free carriers, optical switching can be performed. We demonstrate by computer simulation the optical and electrical characteristics of the devices using a threedimensional (3-D) beam propagation method (BPM) (optical) and the two-dimensional (2-D) device simulation package SILVACO (electrical). The drive current necessary for switching the wavelength in question by one waveguide distance is approximately $0.17 \mathrm{~mA} / \mu \mathrm{m}$ and the attenuation factor is approximately $1 \mathrm{~dB}$. These devices are useful for broad application in both wavelengthdivision multiplexing (WDM) networks and photonics integrated circuits.
\end{abstract}

Index Terms-Arrayed waveguide gratings (AWGs), beam splitter, phase modulation, silicon-on-insulator (SOI), silicon photonics.

\section{INTRODUCTION}

$\mathbf{T}$ HE need for additional functionality and reduction in dimensions has driven research in arrayed waveguide gratings (AWGs) to integrate with various optical components to cope with the emerging increase in data content in optical networks. This creates a strong demand for low-cost reconfigurable optical add-drop multiplexer-demultiplexer (ROADM). The ability to handle a large capacity of wavelengths in an AWG is the key factor in multiple wavelengths routing within the photonic circuitry. Various devices based on AWG for increased functionality have been proposed and demonstrated; one early example is the $4 \times 4$ optical matrix switch for the photonic cell switching at $10 \mathrm{~Gb} / \mathrm{s}$ in silica-based waveguides [1]. Recently, Ito et al. [2] demonstrated an eight-channel AWG for multichannel block switching.

An AWG in silicon-on-insulator (SOI) is of particular interest because of both its technology and material advantages compared to some other technologies, the high index contrast in SOI signifies a small device and better optical confinement within the

Manuscript received October 31, 2005; revised September 14, 2006. The work of F. Y. Gardes was supported by the Engineering and Physical Sciences Research Council (EPSRC), U.K.

S. T. Lim and C. E. Png are with the Institute of High Performance Computing, Agency for Science and Technology, Singapore 117528 (e-mail: limst@ihpc.astar.edu.sg; pngce@ihpc.a-star.edu.sg).

F. Y. Gardes and G. T. Reed are with the Advanced Technology Institute, School of Electronics and Physical Sciences, University of Surrey, Guildford GU2 7XH, U.K. (e-mail: f.gardes@surrey.ac.uk; g.reed@surrey.ac.uk).

Digital Object Identifier 10.1109/JSTQE.2006.885140 waveguide. Ultra-compact devices have been demonstrated in photonic wires [3], [4] but the practicability depends on the quality of the waveguides, in particular the sidewall roughness [5]. In contrast, optical devices based on small cross-sectional SOI rib structures are capable of satisfying both single-mode and zero-birefringence conditions simultaneously [6]. Different SOI optical components have been previously demonstrated, such as phase modulators [7] and AWG [8]. We ask if we can achieve additional functionality by integrating various components and provide optical switching capability.

In this paper, we propose an optically switched SOI AWG by integrating optical phase modulators combined with fourway optical splitters and combiners to reduce the thermo-optic effect (TOE) and decrease the complexity of the device. The SOI AWG demultiplexer has been experimentally demonstrated previously [8], [9]. The wavelengths at the output waveguides are dependent on the optical phase condition. For the case of a wavelength router/switching AWG, implementing optical phase modulators across the grating arms of the AWG can change the optical phase slightly that results in the shift of the wavelength at different output waveguides. However, due to a large number of array waveguides, it is inefficient to implement a phase modulator on each grating arm that could induce undesired TOE. To overcome such a problem, a multiple beam splitter and combiner can be incorporated. This method is compatible with the standard CMOS fabrication processing and no additional processing step is required. We demonstrate an analysis of the device via computer simulation, and illustrate the switching mechanism by integrating a phase modulator. The dc transient and optical characteristics of a low-loss single-mode optical phase modulator based on SOI material are reported. We propose a multiple-beam splitter/combiner using a coupling effect to evenly distribute the optical power into each waveguide arm, thereby reducing the number of phase modulators needed for performing the phase control mechanism.

\section{SOI RIB STRUCTURE}

According to Chan et al. [10], an SOI rib waveguide in its submicron dimensions has to satisfy the following condition in order to achieve single mode and zero birefringence conditions:

$$
\frac{W}{H} \geq 0.05+\frac{(0.92+0.25 H) r}{\sqrt{1-r^{2}}}
$$

where $W$ is the width of the waveguide, $H$ is the guiding layer, and $r$ is the aspect ratio that has been defined in [10].

This condition assumes that high-order vertical modes confined under the rib waveguides were coupled to the outer slab region during propagation, in turn yielding a high propagation 
loss for the higher order modes and maintains its fundamental mode along the propagation distance. The rib waveguide inherently exhibits both majority and minority components that can be shown by the full-vectorial equation expressed in terms of its transverse field components, namely, TE and TM, as

$$
\left[\begin{array}{ll}
P_{x x} & P_{x y} \\
P_{y x} & P_{y y}
\end{array}\right]\left[\begin{array}{l}
E_{x} \\
E_{y}
\end{array}\right]=\beta^{2}\left[\begin{array}{l}
E_{x} \\
E_{y}
\end{array}\right]
$$

where $\beta^{2}$ is the eigenvalue, $E_{x}$ and $E_{y}$ are the electric fields in the $x$ - and $y$-directions, and $P_{x x}, P_{x y}, P_{y x}$, and $P_{x y}$ are the differential operators. This implies that the eigenmodes of such an optical waveguide are usually in a hybrid configuration (both major and minor components co-exist). Due to the small dimension of the rib waveguides proposed in this paper, the minority component cannot be considered as negligible as in the case of large cross-section rib structures [11]. Consequently, the full-vectorial approach is used for the computation of the propagation mode for a waveguide feature size smaller than several micrometers. The presence of the minor component needs to be taken into consideration, since the minority field component has the potential of changing the absolute value of the effective index for a small waveguide cross section. If the waveguide structure is designed such that either the major or minor component is diminutive, then (2) can be approximated as

$$
\begin{aligned}
& P_{x x} E_{x}=\beta_{x}^{2} E_{x} \\
& P_{y y} E_{y}=\beta_{y}^{2} E_{y} .
\end{aligned}
$$

Thus, the transverse field components are decoupled and provide a good approximation for the solution while preserving the dominant polarization effect. The birefringence condition for the polarization-independent waveguide is that the difference between the effective indices of the TE and TM fundamental modes $\left(N_{\mathrm{TE}}-N_{\mathrm{TM}}\right)$ is zero. Full-vectorial computation is conducted to determine single-mode and zero birefringence waveguide geometries. Therefore, polarization is assumed to be negligible in our device.

\section{THEORETICAL MODEL}

Fig. 1 shows a general schematic of an optically switched AWG. The phase condition for the optical field across the grating arms is given as

$$
\Delta \phi=\frac{2 \pi}{\lambda} n_{\mathrm{eff}}(\Delta L+d \sin \theta)
$$

where $n_{\mathrm{eff}}$ is the effective index of the waveguide, $\lambda$ is the center wavelength, $d$ is the array waveguides separation, $\Delta L$ is the path length difference of the array waveguides, and $\theta$ is the dispersion angle that can be calculated by $\sin \left(s_{\text {out }} / R\right)$, where $s_{\text {out }}$ is the output waveguide separation.

In order to perform the cyclic wavelength routing behavior (shifting output wavelength to the adjacent waveguides), the phase condition has to be carefully manipulated by controlling the applied bias on the phase modulator. This manipulated phase condition can be written as

$$
\Delta \phi=\frac{2 \pi}{\lambda} n_{\mathrm{eff}}(d \sin \theta)
$$

From (5), $\Delta L$ will remain constant; hence, the phase condition for switching depends on the small variation in the dispersion angle as calculated in [12]. This will give a phase condition of approximately $\pi / 2 \mathrm{rad}$ needed for the wavelength routing. By substituting the phase condition into (7), the active length $L$ of the phase modulator can be obtained by the carrier concentration. Simplifying

$$
\Delta \phi=\frac{2 \pi \Delta n L}{\lambda}
$$

where $L$ is the active length of the modulator in $z$-direction and $\Delta n$ is the change in the refractive index due to the injected carriers. $\Delta n$, at $\lambda=1.55 \mu \mathrm{m}$, can be determined by [13]

$$
\begin{aligned}
\Delta n & =\Delta n_{e}+\Delta n_{h} \\
& =-8.8 \times 10^{-22}\left(\Delta N_{e}\right)-8.5 \times 10^{-18}\left(\Delta N_{h}\right)^{0.8}
\end{aligned}
$$

where $\Delta n_{e}$ and $\Delta n_{h}$ are the changes in the refractive indexes resulting from the changes in the free electron and hole carrier concentrations, respectively. The changeability in the free carrier concentration will result in a change in the absorption expressed as

$$
\begin{aligned}
\Delta \alpha & =\Delta \alpha_{e}+\Delta \alpha_{h} \\
& =8.5 \times 10^{-18}\left(\Delta N_{e}\right)+6.0 \times 10^{-18}\left(\Delta N_{h}\right)
\end{aligned}
$$

where $\Delta \alpha_{e}$ and $\Delta \alpha_{h}$ are the changes in the absorptions resulting from the changes in the free electron and hole carriers, respectively. According to (5), the phase requirement has to be $2 m \pi$ in order for the optical beams to focus at the respective position at the output plane. It is obvious that if a shift in the output position of the spectrum is required, the phase condition specified by (5) needs to be altered. Thus, to enable the wavelength in question to shift by $q$ (where $q$ is an integer) waveguide distances, it is necessary to create a slight change in the phase across the grating arms for different dispersion angles according to $(6)$.

To perform optical switching, total attenuation of the optical power across the grating arms is needed. This is dependent on the absorption coefficient of (9). Hence, to evaluate the dopant concentration required for switching, the spatial light impinging on the array waveguides has to be resolved. It is assumed that the optical field progression across the star coupler from the input waveguide is Gaussian. This spatial distribution in the focal plane can be obtained by the spatial Fourier transform in the paraxial approximation method [14]. The fundamental mode profile of the center input waveguide can be assumed as a normalized Gaussian power function. The spatial light distribution impinging on the array waveguides is given as [15]

$$
I\left(x_{i}\right)=\sqrt[4]{2 \pi \frac{w_{i}^{2}}{\alpha^{2}}} \exp \left[-\left(\pi w_{i}\left(x_{i} / f\right)\right)^{2}\right]
$$

where $w_{i}$ is the mode field radius with respect to the waveguide in question and $f$ is the focal length product in Fourier optics propagation given in [14]

$$
f=\frac{\lambda R}{n_{s}}
$$




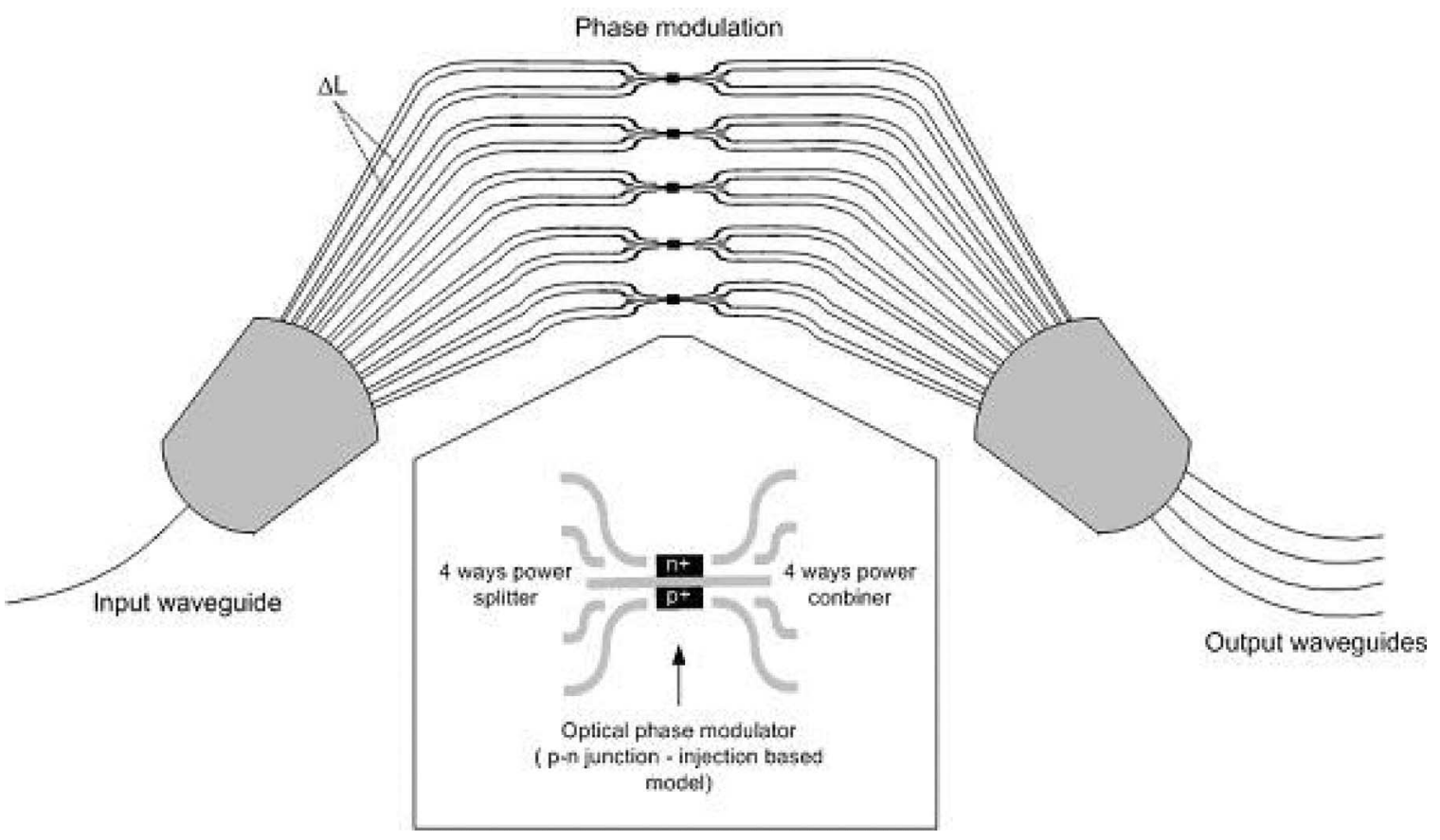

Fig. 1. General schematic of an optically switched AWG. Optical phase modulators are integrated at the mid-section of the grating arms, incorporating a four-way power splitter/combiner.

where $R$ is the focal length and $n_{s}$ is the effective index of the slab. Hence, we obtain the spatial field distribution and focal plane relation. This spatial field distribution will help evaluate the dopant concentration required for the total attenuation for the application of "on/off" switching. The analysis in (10) is sufficient enough for evaluating the normalized field distribution amplitude to create acceptable absorption caused by free carriers, as has been shown previously [8]. However, modeling and simulation of the entire AWG should employ more accurate simulation techniques, such as in [16].

\section{A. $A W G$}

The AWG is designed according to the approach of Amersfoot [17]. The AWG is configured as a $1 \times 4$ demultiplexer with array waveguides and output waveguides separation set at 2.5 and $5 \mu \mathrm{m}$, respectively. The focal length is $104.01 \mu \mathrm{m}$ and the path length difference $\Delta L$ is $18.62 \mu \mathrm{m}$. These equate to a channel spacing $\Delta \lambda$ of $10 \mathrm{~nm}$ and a free spectral range (FSR) of $40 \mathrm{~nm}$. Such a definition will demonstrate the AWG cyclic properties, which provides important additional functionality compared to the simple multiplexers or demultiplexers and plays a key role in more complex devices.

The simulated transmission spectra of the $1 \times 4$ AWG demultiplexer are shown in Fig. 2. The response indicates a cyclic property, where the spectra is repeated every $40 \mathrm{~nm}$ that corresponds to the FSR of AWG. Fig. 3(a)-(d) shows the transmission spectra of four-channel AWG plotted against the image plane distance. Fig. 3(a) illustrates the spectra response of the AWG without any phase alteration. It can be noted that the wavelengths $\lambda_{1}$ and $\lambda_{4}$ will focus at a distance of $\pm 7.5 \mu \mathrm{m}$ away from the origin and $\lambda_{2}$ and $\lambda_{3}$ will focus at $\pm 2.5 \mu \mathrm{m}$, which

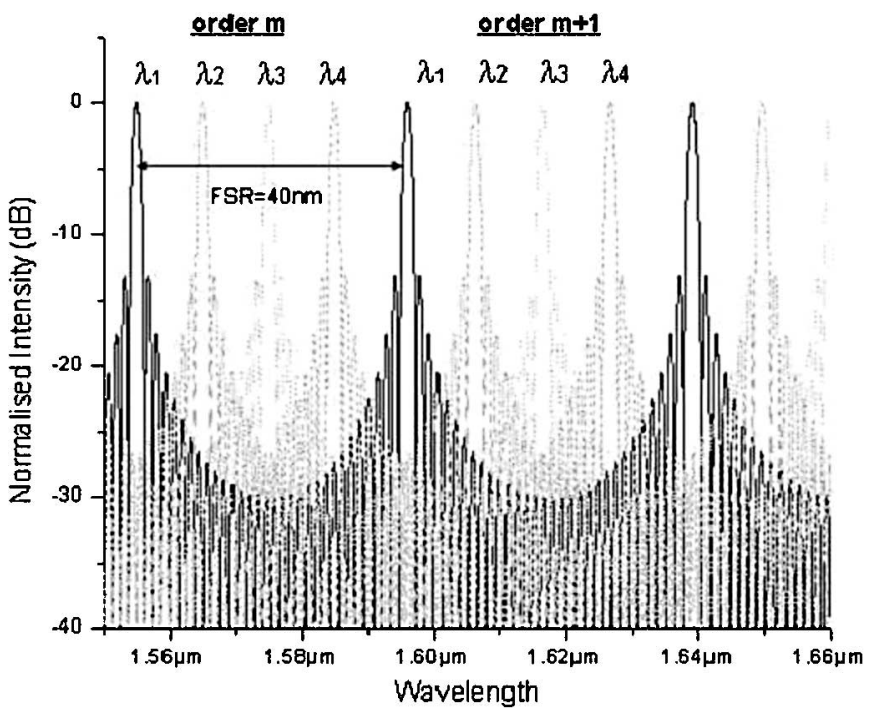

Fig. 2. Simulated response of a $1 \times 4$ AWG demultiplexer at order $m$ and $m+1$; the response displayed periodicity and after each $2 \pi$ change in $\Delta \phi$, the field is repeated.

corresponds to the two inner output waveguide positions. If the phase condition is implemented for different dispersion angles according to the output waveguide locations, then each of the transmission spectra will focus at a position corresponding to the appropriate output waveguides. Hence, for dispersion angles of $2.75^{\circ}, 5.5^{\circ}$, and $8.25^{\circ}$, the spectra will shift by distances of 5,10 , and $15 \mu \mathrm{m}$, respectively, as shown in Fig. 3(b)-(d). The shift in the distance indicates the respective output waveguide locations where waveguide separation is designed at $5 \mu \mathrm{m}$. This displays a potential capability for simultaneous wavelength switching. 


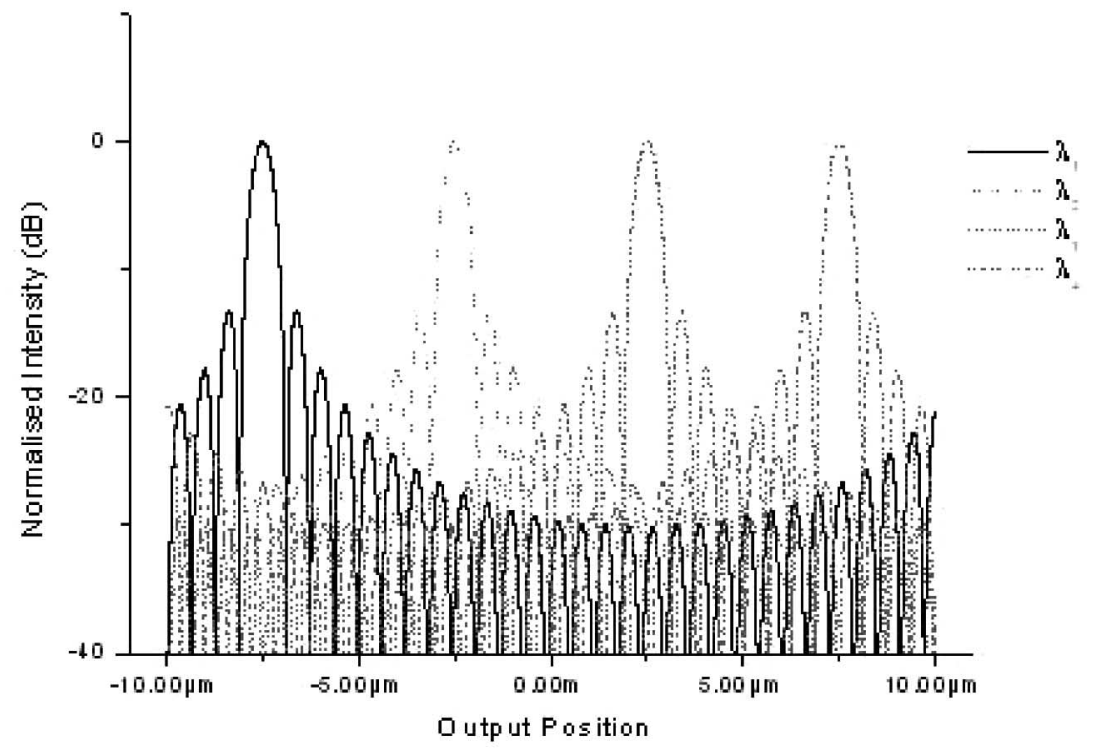

(a)

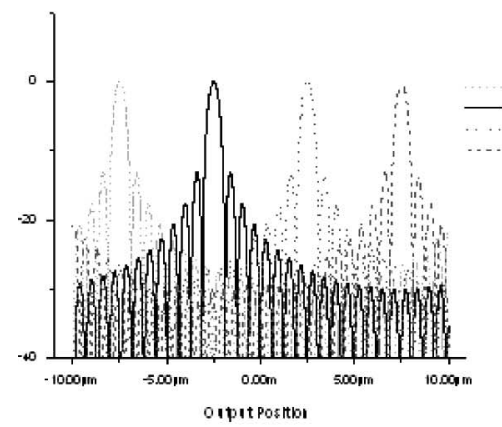

(b)

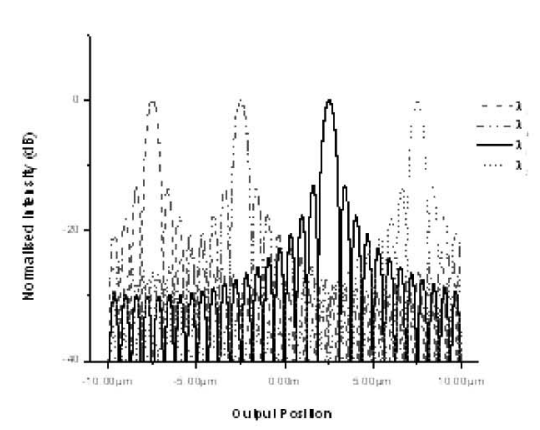

(c)

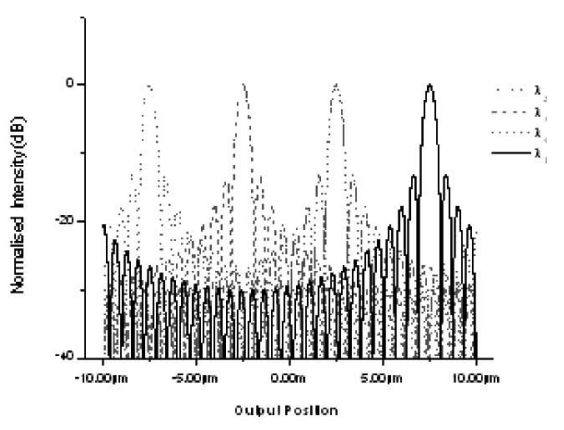

(d)

Fig. 3. Simulated responses of the optically switched AWG. (a) Conventional AWG without any phase alteration. (b) Response shifted by a waveguide distance $\left(S_{\text {out }}=5 \mu \mathrm{m}, \Delta \phi=\pi / 2\right.$ ). (c) Response shifted by a waveguide distance ( $S_{\text {out }}=10 \mu \mathrm{m}, \Delta \phi=\pi$ ). (d) Response shifted by a waveguide distance $\left(S_{\text {out }}=15 \mu \mathrm{m}, \Delta \phi=3 \pi / 2\right)$.

\section{B. Optical Phase Modulator}

1) Device Structure: The device structure of the phase modulator is shown in Fig. 4. It is a lateral optical phase modulator integrated into a low-loss SOI rib waveguide. The device is a two-terminal $\mathrm{p}$ - $\mathrm{i}-\mathrm{n}$ structure where both $\mathrm{n}$ and $\mathrm{p}$ regions were modeled as highly doped regions with constant doping concentrations of $10^{20} \mathrm{~cm}^{-3}$, based around an overall silicon thickness of $1 \mu \mathrm{m}$ with etched rib waveguides $0.49-\mu \mathrm{m}$ wide and an etch depth of $0.65 \mu \mathrm{m}$. They are predicted to exhibit single-mode operation and zero birefringence [10]. Furthermore, oxide isolation trenches are employed on either side of the doping regions to improve injection efficiency, following Hewitt et al. [14].

2) Electrical and Optical Models: The two-dimensional (2-D) ATLAS device simulation package from SILVACO [18] has been used to predict the dc and transient characteristics of the modulator. The simulator numerically predicts internal physics and device characteristics of semiconductor devices by solving Poisson's equation and the charge continuity equations for holes and electrons. The software allows a complete statistical approach (Fermi-Dirac statistics) when instances such as heavily doped regions are considered. Shockley-Reed-Hall

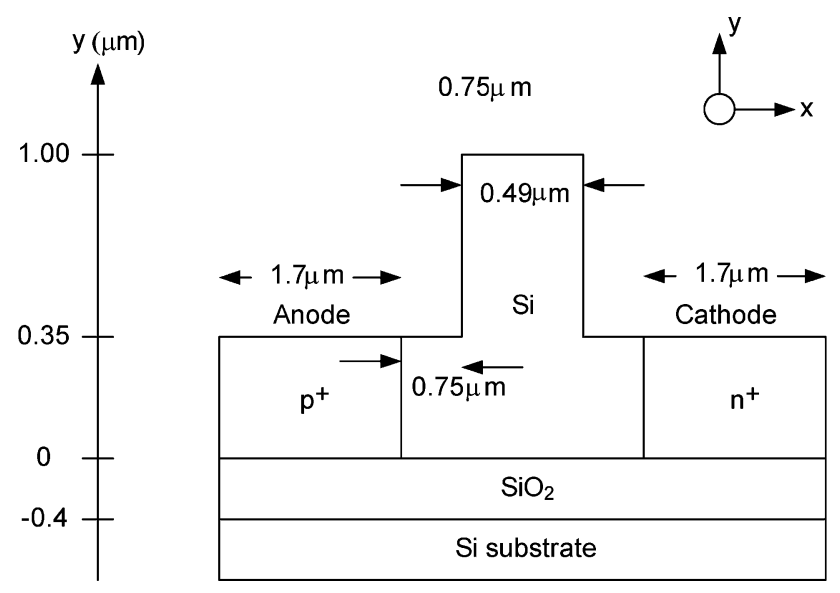

Fig. 4. Geometry of the proposed two-terminal p-i-n optical phase modulator.

(SRH), Auger, and surface recombination models were included to account for the carrier recombination. A carrier concentration dependent SRH recombination model was employed, with an estimated carrier lifetime in the intrinsic Si device layer 
TABLE I

Simulation PARAMETER SETTINGS AT $300 \mathrm{~K}$

\begin{tabular}{cc}
\hline \hline$\lambda(\mu \mathrm{m})$ & 1.55 \\
\hline $\mathrm{Si}$ refractive index & 3.47 \\
$\mathrm{SiO}_{2}$ refractive index & 1.44 \\
Electron carrier lifetime (ns) & 700 \\
Hole carrier lifetime (ns) & 300 \\
Si background carrier concentration (atoms $\left./ \mathrm{cm}^{3}\right)$ & $10^{15}$ \\
\hline \hline
\end{tabular}

(concentration of $10^{15} \mathrm{~cm}^{-3}$ ) of electrons and holes with $\tau_{n}=700 \mathrm{~ns}$ and $\tau_{p}=300 \mathrm{~ns}$, respectively. Contact pads were assumed to be ohmic in nature and, hence, carry no additional contact resistance or capacitance. The main parameters used in the simulations are listed in Table I. From the dc and transient simulations, ATLAS calculates the injected free carrier concentrations in the intrinsic region of the devices for both dc and transient biasing conditions. The change in the concentration of free carriers is then converted to the refractive index change in the device by using (8). This approach has been validated in the past by various authors (e.g., [7], [19]-[21]). In order to maximize this change, we must optimize the interaction between the injected free carriers and the propagating optical mode.

Following the work of Png et al. [7], it was determined that a high degree of uniformity exists in the injected carrier concentration at the levels of interest throughout the central guiding region of the device. This results in a uniform refractive index change across the waveguiding region. After obtaining the mean value of the injected carrier concentration in the guiding region, we applied the results to (8) and (9) to obtain the resulting changes in the refractive index and absorption for the device under investigation at a wavelength of $\lambda=1.55 \mu \mathrm{m}$. Alternatively, the data from the electrical simulation could be transferred to a similar grid-based optical simulator to predict absorption and refractive index changes, but due to the uniform nature of the injected charge there should be negligible difference between these approaches.

The proposed modulator structure in Fig. 4 was not shown to have any surface passivation. In actual fabrication and deployment, this is not the case as the passivation oxide is required to:

1) provide protection for the physical integrity of the optical waveguide structure;

2) allow metal connections to be made for subsequent electrical connection;

3) act as insulation between metal contacts for different contact regions;

4) reduce surface recombination at the silicon interface.

A likely candidate for the surface passivation could be thermally grown silicon dioxide (e.g., [21], [22]). Due to the abrupt discontinuity of the lattice structure at the surface, a large number of localized energy states or generation-recombination centers may be introduced at the surface region [23]. In our case, we considered the interface recombination in our simulation model with a recombination speed of $100 \mathrm{~cm} / \mathrm{s}$ that corresponds to thermally grown $\mathrm{SiO}_{2}$, as is typical in an actual fabrication scenario.

The three-dimensional (3-D) beam propagation method (BPM) was used for calculating the modal field profile and optical losses due to carrier absorption. From the values of the electron and hole concentrations at any point of the $p-i-n$

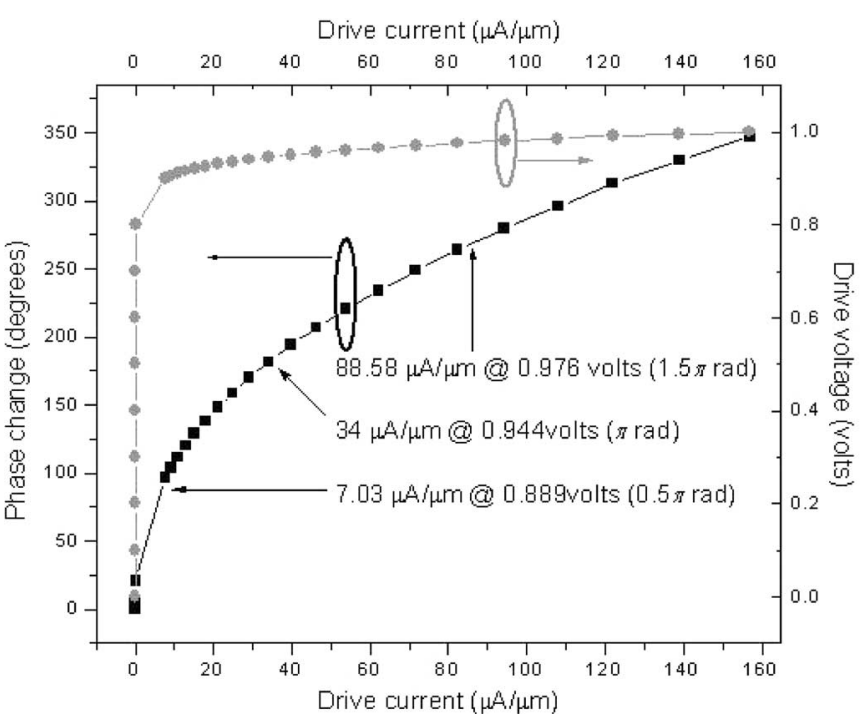

Fig. 5. Phase modulation against drive current and its associated $I-V$ characteristics.

structure, which were determined using the electrical model described earlier, the induced real refractive index change $(\Delta n)$ and the optical absorption change $(\Delta \alpha)$ produced by the free carrier dispersion (highly doped and injected carrier regions) at $\lambda=1.55 \mu \mathrm{m}$ can be determined by (8) and (9), respectively. Also, the change in the refractive index results in a phase shift $\Delta \phi$ in the optical mode can be approximated by (7). Thus, we can rearrange (7) to solve for $\Delta n$ and determine the required refractive index change and, hence, the required carrier density to achieve the desired amount of phase shift for a certain device length or vice versa. Unless otherwise stated, the active modulator length is assumed to be $500 \mu \mathrm{m}$.

3) Result and Discussion: From Fig. 5, the change in the refractive index and, hence, phase change varies nonlinearly with the applied current (black line). One factor that contributes to the nonlinearity of the change in the phase versus current density relation is the sublinear dependence of the change in the free holes, $\Delta N_{h}$ with the change in the refractive index, as shown in (8). Also, as the modulator is driven harder, more free carriers are injected into the intrinsic region of the device. This increase in the concentration of the previously intrinsic region results in an increase in the Auger recombination rate (at the injected carrier concentrations much greater than $10^{17} \mathrm{~cm}^{-3}$, the Auger recombination becomes the dominant recombination process, e.g., [19]). This results in a reduced lifetime in this region and, hence, we have to drive the modulator harder to achieve an equivalent refractive index change than at lower drive powers. Of course, an increase in the recombination rate will result in a faster switching device, i.e., reduction in the rise and fall times of the modulator. The associated $I-V$ characteristics of the optical diode (gray line) are included. Of special interests are the bias points required to obtain increments of $\pi / 2 \mathrm{rad}$ for implementation into the proposed AWG. The current required to obtain $\pi / 2-, \pi-$, and $3 \pi / 2-\mathrm{rad}$ phase shifts are $7.03 \mu \mathrm{A} / \mu \mathrm{m}$ $(0.889 \mathrm{~V}), 34 \mu \mathrm{A} / \mu \mathrm{m}(0.944 \mathrm{~V})$, and $88.58 \mu \mathrm{A} / \mu \mathrm{m}(0.976 \mathrm{~V})$, respectively. 


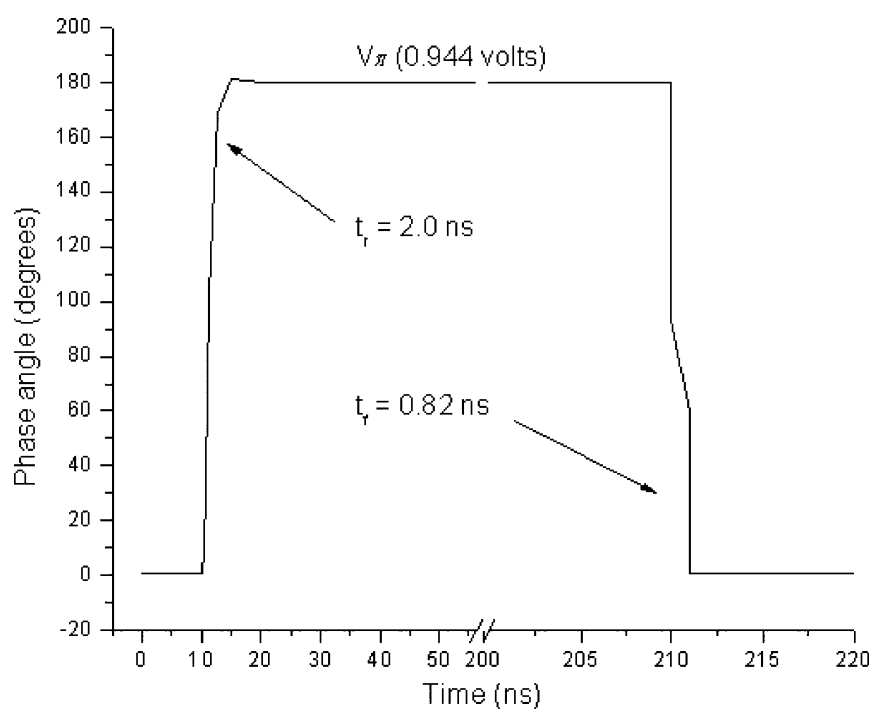

Fig. 6. Phase modulation versus transient time. The rise time is the limiting transition and, hence, determines the device operating speed.

A transient modeling solution was employed. Both anode and cathode were first zero biased for $10 \mathrm{~ns}$, followed by a step increase to $V_{\pi}$ for $200 \mathrm{~ns}$, and a subsequent step decrease to 0 V. $V_{\pi}$ is the voltage corresponding to $180^{\circ}$ phase shift. The rise time $t_{r}$ is defined as the time required for the induced phase shift to change from $10 \%$ to $90 \%$ of the maximum value. Likewise, the fall time $t_{f}$ is defined as the time required for the induced phase shift to change from $90 \%$ to $10 \%$ of the maximum value. For the modulator shown in Fig. 4, the rise and fall times were determined to be $t_{r}=2.0 \mathrm{~ns}$ and $t_{f}=0.82 \mathrm{~ns}$, respectively.

Fig. 6 shows the rise and fall times and it is clear that the rise time is the slower of the two and, hence, the limiting transition. In order to clearly illustrate both the rise and fall times, a "break" in the horizontal time axis was made so that the rising and falling edges of the transient waveform could be highlighted.

The doped regions were positioned far from the rib $(0.75 \mu \mathrm{m})$ so that the significant overlap with the optical mode is minimized, resulting in approximately $0.1 \mathrm{~dB}$ of passive optical loss. The price for this low loss translates to a slow device speed. This is necessary in order to ease implementation into AWG devices and minimize optical attenuation.

The active optical absorption is defined as the device optical propagation loss at various switching bias points of interest and is attributed to the additional absorption loss due to the injected electrons and holes. The number of injected carriers in the waveguiding region is $2 \times 10^{17}, 4.53 \times 10^{17}$, and $7.2 \times 10^{17} \mathrm{~cm}^{-3}$ for switching at $0.5 \pi, \pi$, and $1.5 \pi$ rad, respectively. In turn, the absorption coefficient $\alpha$ is $2.9^{-}, 6.6^{-}$, and $10.4 \mathrm{~cm}^{-1}$, respectively (i.e., $\sim 12.6, \sim 28.5$, and $\sim 45.34 \mathrm{~dB} / \mathrm{cm}$, respectively).

It should be noted that the modulator device speed reported here is rather modest and nowhere near one of the fastest reported recently [22]. Nonetheless, the device switching performance can be improved without changing its physical dimensions by overdriving during the device rise and fall times [7]. This method was recently employed by $\mathrm{Xu}$ et al. [23] to over-

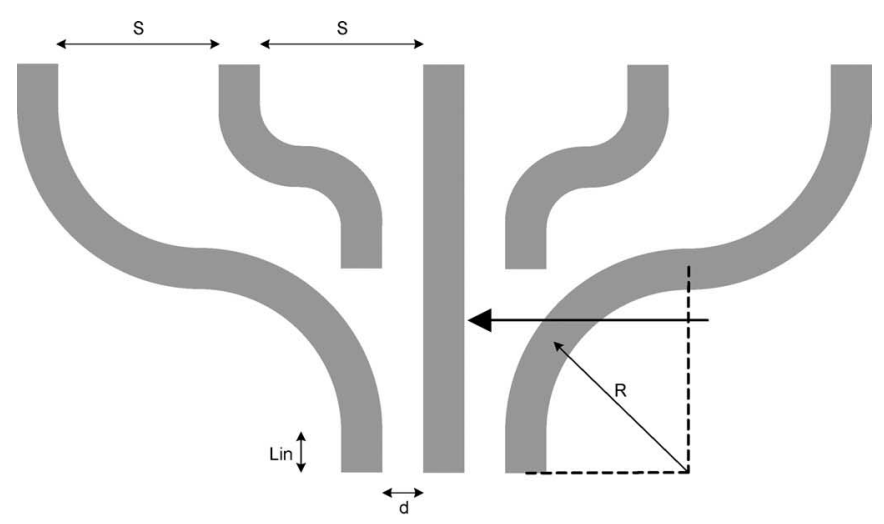

(a)

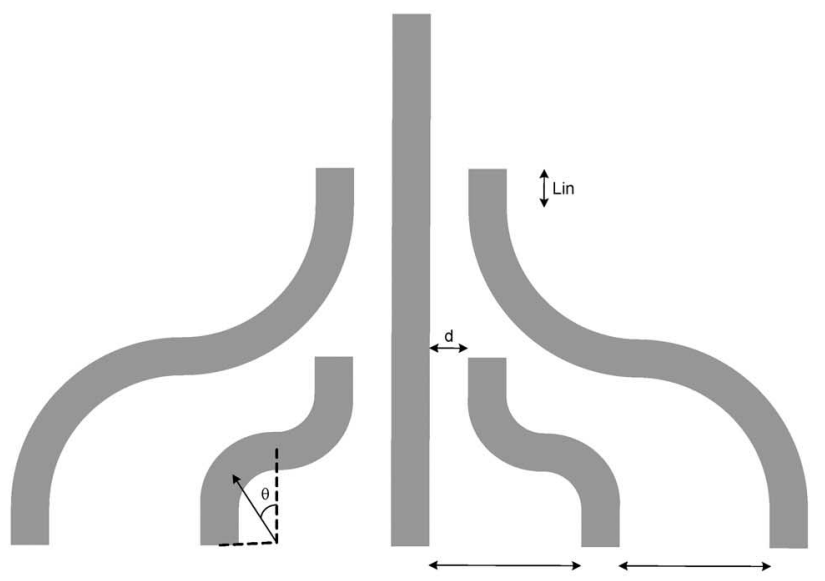

(b)

Fig. 7. (a) Multiple beam splitter. (b) Multiple beam combiner.

drive a strip-based ring resonator waveguide to operate at a data rate of $1.5 \mathrm{~Gb} / \mathrm{s}$.

\section{Multiple Beam Splitters}

Fig. 7 shows the schematic of the four-way beam combiner/splitter using waveguide couplers, which will be implemented across the grating section of the AWG. The beam combiner/splitter consists of a straight waveguide, which acts as the modulating arm and four S-bends. Each of the S-bends comprises a variable input section $L_{\mathrm{in}}$, and the length of $L_{\mathrm{in}}$ depends on the waveguide separation $d$.

The total length of the S-bend is given by

$$
L_{\text {S-bend }}=2 L_{\text {in }}+\left(\frac{2 \pi \theta_{\text {bend }} R}{360}\right)
$$

where $\theta_{\text {bend }}$ is the angle and $R$ is the radius of the bend, respectively.

The grating arms are arranged in groups of four in this particular case, and the optical phase modulator is located at the center of the straight waveguide. The optical power coupled from the free space region to the array waveguides will be combined to the straight waveguide where phase manipulation occurs after which the optical power will be split equally to the array waveguides in question. The phase condition of $2 m \pi$ is still needed for constructive interference at the second slab region, which 

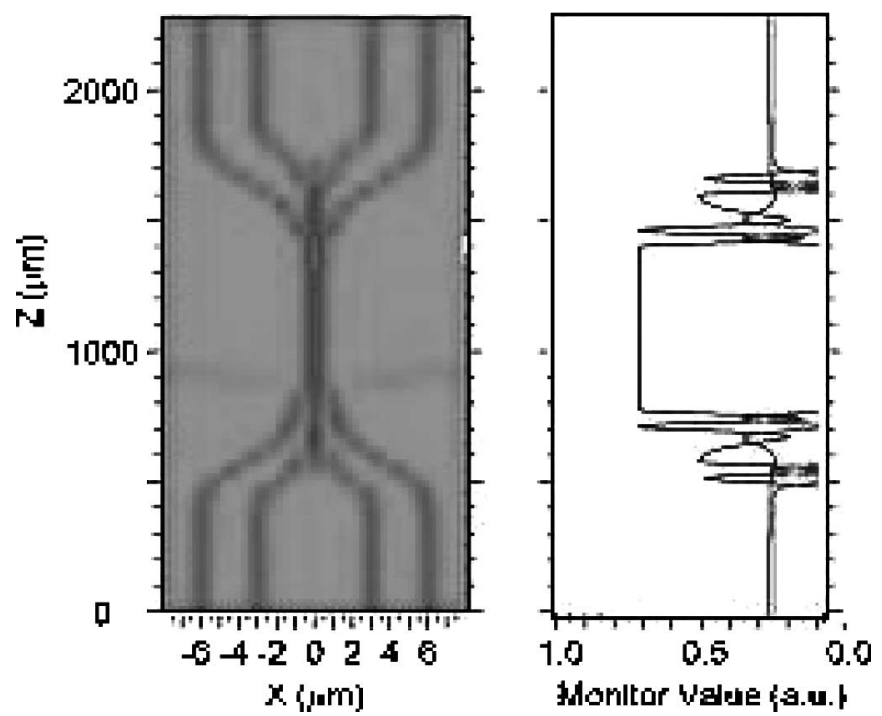

Fig. 8. Simulated result of the multiple beam splitter and combiner with 1.5-dB excess loss.

can be easily compensated for by adding the optical path length within the grating arms. The splitter is modeled using the 3-D BPM propagation method. The simulation only considered TE polarization and assumed the rib structure to be polarizationindependent. For a combiner, an inverted symmetrical configuration is proposed and it is assumed that the optical operation is similar to that of a splitter with the appropriate optimization necessary for the length $L_{\text {in }}$.

The output separation $s$ is $2.5 \mu \mathrm{m}$ where coupling will be negligible and the $\mathrm{S}$-bends are placed at $0.4 \mu \mathrm{m}$ distance away from their neighbors. Fig. 8 shows the simulated results for the proposed splitter/combiner, and it can be observed that the optical power is equally distributed across the four S-bends with each waveguide receiving a quarter of the optical power, combined at a later stage. Excess loss of the splitter and combiner is estimated to be 1.5-dB that is an acceptable benchmark. The straight waveguide acts as a modulation arm where the phase of the optical mode will be altered according to the phase requirement, as indicated in Section III-B.

\section{CONCLUSION}

The demand for the high functionality in AWG has lead to the investigation of simultaneous optical switching to ensure flexibility and survivability of the optical systems. The factors that probably dominate such phenomenon are integrations of both active and passive optical components within the AWG for supporting diverse applications. The trend to device miniaturization also fuels the need to use high index contrast material, such as the SOI, to produce smaller structures and better optical confinement.

We have demonstrated the cyclic property of AWG, which is useful for optical switching, by deploying a phase modulation mechanism within the grating region. Such a mechanism can be analyzed using the 2-D semiconductor simulation package SILVACO to investigate the device characteristics, namely the dc and transient behavior of the active region of the modulator. The proposed device also includes multiple beam splitters/combiners to facilitate the modulation functionality of the optical mode within the array waveguides, hence, reducing the number of phase modulators required, and simplifies fabrication.

\section{REFERENCES}

[1] J. Sasaki, H. Hatakeyama, T. Tamanuki, S. Kitamura, M. Yamaguchi, N. Kitamura, T. Shimoda, M. Kitamura, T. Kato, and M. Itoh, "Hybrid integrated $4 \times 4$ optical matrix switch using self-aligned semiconductor optical amplifier gate arrays and silica planar lightwave circuits," Electron. Lett., vol. 34, no. 10, pp. 986-987, May 1998.

[2] T. Ito, I. Ogawa, Y. Suzaki, K. Magari, Y. Kawaguchi, Y. Suzuki, and N. Ishihara, "Eight-channel simultaneous wavelength conversion from equal to unequal channel spacing," IEEE Photon. Technol. Lett., vol. 13 no. 10, pp. 1106-1108, Oct. 2001.

[3] K. Sasaki, F. Ohno, A. Motegi, and T. Baba, "Arrayed waveguide grating of $70 \times 60 \mu \mathrm{m}^{2}$ size based on Si photonic wire waveguides," Electron. Lett., vol. 14, no. 14, pp. 801-802, 2005.

[4] P. Dumon, W. Bogaerts, D. Van Thourhout, D. Taillaert, V. Wiauz, S. Beckx, J. Wouters, and R. Baets, "Wavelength-selective components in SOI photonics wires fabricated with deep UV lithography," in Proc. Group IV Photon., Kowloon, Hong Kong, p. WB5, 2004.

[5] F. Grillot, L. Vivien, S. Laval, and E. Cassan, "Propagation loss in single-mode ultrasmall square silicon-on-insulator optical waveguides," J. Lightw. Technol., vol. 24, no. 2, pp. 891-895, Feb. 2006.

[6] W. R. Headley, G. T. Reed, S. Howe, A. Liu, and M. Paniccia, "Polarisation independent optical racetrack resonators in silicon on insulator," Appl. Phys. Lett., vol. 85, no. 23, pp. 5523-5525, 2004.

[7] C. E. Png, S. P. Chan, S. T. Lim, and G. T. Reed, "Optical phase modulators for $\mathrm{MHz}$ and $\mathrm{GHz}$ modulation in silicon-on-insulator (SOI)," J. Lightw. Technol., vol. 22, no. 6, pp. 1573-1582, Jun. 2004.

[8] S. T. Lim, C. E. Png, S. P. Chan, and G. T. Reed, "Flat spectral response silicon arrayed waveguide gratings via ion implantation," Opt. Express, vol. 14, no. 14, pp. 6469-6478, 2006.

[9] M. R. T. Person, A. Bezinger, A. Delage, J. W. Fraser, S. Janz, P. E. Jessop, and D.-X. Xu, "Arrayed waveguide grating demultiplexers in silicon-oninsulator," Proc. SPIE, vol. 2953, pp. 11-18, 2004.

[10] S. P. Chan, C. E. Png, S. T. Lim, G. T. Reed, and V. M. N. Passaro, "Single mode and polarisation independent silicon-on-insulator waveguides with small cross section," J. Lightw. Technol., vol. 23, no. 6, pp. 2103-2111, Jun. 2005.

[11] J. Lousteau, D. Furniss, A. Seddon, T. M. Benson, A. Vukovic, and P. Sewell, "The single mode conditionfor silicon-on-insulator optical rib waveguides with large cross section," J. Lightw. Technol., vol. 22, no. 8, pp. 1923-1929, Aug. 2004.

[12] M. K. Smit and C. V. Dam, "Phasar-based WDM-devices: Principle, design and applications," IEEE J. Sel. Topics Quantum Electron., vol. 2, no. 2, pp. 236-250, Jun. 1996.

[13] R. A. Soref and B. R. Bennett, "Electrooptical effects in silicon," IEEE J. Quantum Electron., vol. QE-23, no. 1, pp. 123-129, Jan. 1987.

[14] J. W. Goodman, "Introduction to Fourier optics," in Classic Textbook Reissue Series. New York: McGraw-Hill, 1998, ch. 5, pp. 83-90.

[15] P. Munoz, D. Pastor, and J. Capmany, "Modeling and design of arrayed waveguide gratings," J. Lightw. Technol., vol. 20, no. 4, pp. 661-674, Apr. 2002.

[16] D. X. Dai and S. L. He, "Accurate two-dimensional model of an arrayed waveguide grating demultiplexer and optimal design based in the reciprocity theory," J. Opt. Soc. Amer. A, Opt. Image Sci., vol. 21, no. 12, pp. 2392-2398, 2004.

[17] M. Amersfoort, "Arrayed waveguide grating: Design, simulation and layout using Prometheus DV," Appl. Note A1998003, Kymata, Enschede, The Netherlands, Jun. 1998.

[18] ATLAS User's Manual, SILVACO International, Santa Clara, CA, 2005.

[19] C. K. Tang, G. T. Reed, A. J. Walton, and A. G. Rickman, "Low-loss, single-mode, optical phase modulator in SIMOX material," J. Lightw. Technol., vol. 12, no. 8, pp. 1394-1400, Aug. 1994.

[20] P. D. Hewitt and G. T. Reed, "Improved modulation performance of a silicon p-i-n device by trench isolation," J. Lightw. Technol., vol. 19, no. 3, pp. 387-390, Mar. 2001.

[21] C. A. Barrios and M. Lipson, "Modeling and analysis of highspeed electro-optic modulation in high confinement silicon waveguides 
using metal-oxide-semiconductor configuration,"J. Appl. Phys., vol. 96, pp. 6008-6015, 2004.

[22] L. Liao, D. Samara-Rubio, M. Morse, A. Liu, D. Hodge, D. Rubin, U. D. Keil, and T. Franck, "High speed silicon Mach-Zehnder modulator," Opt. Express, vol. 13, pp. 3129-3135, 2005.

[23] Q. Xu, B. Schmidt, S. Pradhan, and M. Lipson, "Micrometre-scale silicon electro-optic modulator," Nature, vol. 435, pp. 325-327, 2005.

Soon Thor Lim was born in Singapore in 1975. He received the electrical and electronics degree (with honors) and the Ph.D. degree in physics from the University of Surrey, Guildford, U.K., in 2001 and 2005, respectively.

Currently, he is with the Institute of High Performance Computing, Agency for Science and Technology, Singapore. His current research interests include SOI waveguides, photonic crystals, and photonics bio-sensing applications.

Ching Eng Png was born in Singapore in 1974. He received the B.Eng. degree (with honors) in electronic and electrical engineering and the Ph.D. degree in silicon photonics from the University of Surrey, Guildford, U.K., in 1999 and 2004 , respectively.

From November 1999 to September 2000, he was a Gigabit Optical Transceiver Engineer at Agilent Technologies, Singapore. Currently, he is with the Institute of High Performance Computing, Agency for Science and Technology, Singapore, where he is engaged in research in the field of bio-photonics and silicon photonics.

Dr. Png was the recipient of the Royal Academy of Engineering Prize and the Institution of Electrical Engineers (IEE) Hudswell International Research Scholarship for his work on silicon optical modulators.
Frederic Y. Gardes was born in France in 1977. He received the D.U.T. degree in physics measures from the University Institute of Technology (IUT) of Lannion, Lannion, France, in 1999, the B.Sc. degree (with honors) in applied physics from the University of Portsmouth, Portsmouth, U.K., in 2001, and the M.Sc. degree in optoelectronics from the University of Northumbria, Newcastle upon Tyne, U.K., in 2002. Currently, he is working toward the Ph.D. degree at the Advanced Technology Institute, School of Electronics and Physical Sciences, University of Surrey, Guildford, U.K.

His current research interests include integrated optics in silicon-on-insulator (SOI), numerical simulation of optical and semiconductor components, optical modulators based on the plasma dispersion effect, and ring resonators.

Graham T. Reed received the B.Sc. degree (with honours) in electronics and electrical engineering and the Ph.D. degree from the University of Surrey, Guildford, U.K., in 1983 and 1987, respectively.

He joined the University of Surrey, Guildford, U.K., in 1989 with the aim of establishing a research activity in guided wave optoelectronics, and now leads an internationally recognized group. He is responsible for initiating a new research field in the U.K. on silicon integrated optical circuits, and his group has produced a series of leading technical advances in the field worldwide, notably in optical modulators, grating couplers, and optical sensing applications. A testament to the originality and potential of the silicon work is that Bookham Technology Public Limited Company adopted it as their core business in the early 1990s. His work is built upon collaborative arrangements with both industry and academia alike. His work has been associated with companies and universities in the U.K., the U.S., France, Italy, Germany, Japan, and Singapore. $\mathrm{He}$ is the author or coauthor of more than 150 publications in the field of guided wave optoelectronics. He has coauthored the first textbook on silicon photonics and is a holder of several patents.

Prof. Reed is a Fellow of the Institution of Electrical Engineers, U.K. 